\title{
Acknowledgement to Reviewers
}

The editor-in-chief extends her appreciation to the editorial board members and to all ad hoc reviewers whose comments and criticisms ensure the timeliness and quality of the papers published in this journal. We are especially grateful to those members of the editorial board who, after serving for many years, have retired from the board, and we look forward to the new colleagues who will join us. Finally, we thank all of you, authors and contributors, who give this journal the international standing in the research into dementing disorders that it enjoys.

Dag Aarsland

Liesbeth Aerts

Alia Algwiri

Suvarna Alladi

Nadeshda Andrejeva

David L. Bachman

Nancy Baenziger

B. Lynn Beattie

Ladina Bezzola

Imrich Blasko

Marina Boban

Laura Bonanni

Kolbjørn S. Bronnick

Gerard Byrne

Francisco Felix Caballero

Richard Camicioli

Barbara Caracciolo

Somnath Chatterji

Sarah Cook

Andrea Corsonello

Diogo Costa

Sam Creavin

Emma L. Cunningham

Lila Davachi

Luis Fernando de Araujo

Alexandre de Mendonça

Martin Dichter

Stefano Diciotti

Breno Diniz

Warren Donnellan

Bruno Dubois

Knut Engedal

Thierry Ettlin

\author{
Rosemary Fama \\ Martin Farlow \\ Bente Finsen \\ Majid Fotuhi \\ Robert P. Friedland \\ Toshiya Fukui
}

Samuel Gandy

Ravindra Garg

Valentina Garibotto

Ellen Gelpi

Amitabha Ghosh

Gabriel Gold

Gabriel Gonzalez-Escamilla

Milica G. Gregoric Kramberger

Kasia Gustaw Rothenberg

Kirsten Hall Long

Yvonne Han

Robert Hoerr

Ramona Hopkins

Ging-Yuek R. Hsiung

Wen-Long $\mathrm{Hu}$

Edward D. Huey

Bettina Husebo

Kristina Riis Iden

Kate Irving

Willemijn Jansen

Kurt A. Jellinger

Frank Jessen

Hanna Jokinen

Matthew Jones

Yogeshwar Kalkonde

Dan Kaufer 
Dementia

and Geriatric
Cognitive Disorders
Dement Geriatr Cogn Disord 2016;42:369-370

DOI: 10.1159/000453055

Acknowledgement to Reviewers

SangYun Kim

Juliet King

Anne-Brita A. Knapskog

Shinsuke Koike

Carsten Korth

Zuzanna Kurowska

Pinar Kurt

Timothy Kwok

Richard Lathe

Hochang B. Lee

Min Li

Bjorn Eric Neerland

Rónán O'Caoimh

Cynthia Olsen

Chiadi U. Onyike

Nancy Pachana

Ming-Chyi Pai

George P. Paraskevas

Marian B. Patterson

Karalyn Patterson

Miguel Á. Pérez

George Perry

Ramit Ravona-Springer

Gustavo C. Roman

Ingmar Rosen

Hanna Rosenmann
Prasun Roy

Seon Young Ryu

Amanda Sacker

Seyed Sajjadi

David P. Salmon

Elizabeth Sampson

Pascual Sanchez-Juan

Philip Scheltens

Paul E. Schulz

Timothy Shepherd

Nobuto Shibata

Shireen Sindi

Vegard Skirbekk

Pietro Spataro

David Steffens

Janine Stein

Christopher Stewart

Andrzej Szutowicz

T. Takahashi

Achille Tchalla

Edmond Teng

Tim Vanbellingen

Anders Wallin

Henry Weinstein

Ignaz Wessler

Dan Wilson

Claire A. Wolfs 